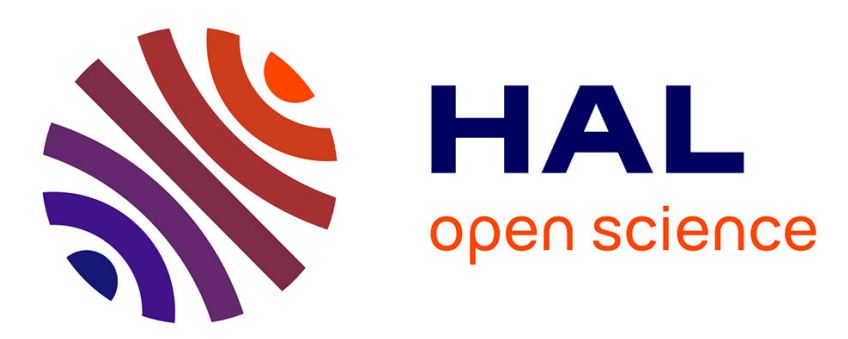

\title{
Predictor-based sampled-data exponential stabilization through continuous-discrete observers
}

Frédéric Mazenc, Emilia Fridman

\section{To cite this version:}

Frédéric Mazenc, Emilia Fridman. Predictor-based sampled-data exponential stabilization through continuous-discrete observers. Automatica, 2016, 63, pp.7. 10.1016/j.automatica.2015.10.016 . hal01248072

\section{HAL Id: hal-01248072 \\ https://inria.hal.science/hal-01248072}

Submitted on 23 Dec 2015

HAL is a multi-disciplinary open access archive for the deposit and dissemination of scientific research documents, whether they are published or not. The documents may come from teaching and research institutions in France or abroad, or from public or private research centers.
L'archive ouverte pluridisciplinaire HAL, est destinée au dépôt et à la diffusion de documents scientifiques de niveau recherche, publiés ou non, émanant des établissements d'enseignement et de recherche français ou étrangers, des laboratoires publics ou privés. 


\title{
Predictor-based sampled-data exponential stabilization through continuous-discrete observers*
}

\author{
Frédéric Mazenc $^{\mathrm{a}}$, and Emilia Fridman ${ }^{\mathrm{b}}$ \\ ${ }^{a}$ EPI DISCO Inria-Saclay, Laboratoire des Signaux et Systèmes (L2S, UMR CNRS 8506), CNRS, CentraleSupélec, Université \\ Paris-Sud, 3 rue Joliot Curie, 91192, Gif-sur-Yvette, France. \\ ${ }^{\mathrm{b}}$ School of Electrical Engineering, Tel Aviv University, 69974, Israel.
}

\begin{abstract}
The problem of stabilizing a linear continuous-time system with discrete-time measurements and a sampled input with a pointwise constant delay is considered. In a first part, we design a continuous-discrete observer which converges when the maximum time interval between two consecutive measurements is sufficiently small. In a second part, we construct a dynamic output feedback by using a technique which is strongly reminiscent of the reduction model approach. It stabilizes the system when the maximal time between two consecutive sampling instants is sufficiently small. No limitation on the size of the delay is imposed and an ISS property with respect to additive disturbances is established.
\end{abstract}

Key words: Continuous-discrete observer, delay, stabilization.

\section{Introduction}

For linear, possibly time-varying, systems with continuous measurements with a delayed input and no sampling, there are in the literature several stabilization techniques. Some of them are predictor based techniques and some of them are variants of the reduction model approach. The main advantage of the latter is to provide with globally asymptotically stabilizing control laws for systems with an arbitrarily large pointwise or distributed delay in the input. This technique has been developed in particular in Mayne (1968), Witrant et al. (2007), Artstein (1982) and more recently in Mazenc et al. 2 (2012), Mazenc et al. (2014), Mazenc \& Malisoff (2014) (see also the references therein). Recently, in the contribution Mazenc \& Normand-Cyrot (2013), this control design technique has been adapted to linear systems with piecewise constant inputs, in the case where the entire state is continuously measured. But the problem of applying this technique when only some components of the state variables are measured and the measurements are available at discrete time only is still open. But the motivation for studying it is strong since, in practice, in many cases, the measurements are discrete. In order to solve it, one may try to adapt three classical techniques.

\footnotetext{
* Corresponding author: F. Mazenc. Supported by l'Institut pour le Contrôle et la Décision de l'Idex Paris-Saclay (iCODE) (Mazenc) and partially supported by Israel Science Foundation (grants No 754/10 and No 1128/14) (Fridman).

Email addresses:

frederic.mazenc@12s. centralesupelec.fr (Frédéric

Mazenc), emilia@eng.tau.ac.il (Emilia Fridman).
}

First, when the length of sampling time intervals and of the output measurement intervals is the same and the delay is a multiple of it, the system, in the time-invariant case, can be discretized (see Kazantzis et al. (2005)) and, in a second step, stabilized via the design of an observer and the utilization of the discrete-time version of the reduction model approach as done in Castillo-Toledo et al. (2010), Gonzalez et al. (2012). The second possible strategy consists in applying the reduction model approach in combination with continuous observers. The third possible attempt consists in developing a technique of stabilization based on observers of continuous-discrete type and a new version of the reduction model approach, taking into account the discontinuous aspect of the observer. This is the objective we pursue the present work, which is an extension of the preliminary paper Mazenc \& Fridman (2014). Before describing in more details its main results, let us mention that continuous-discrete observers have been studied for a long time and the seminal papers Jazwinski (1970) and Deza et al. (1992) have inspired many theoretical and applied papers. For more information, the reader is referred in particular to Andrieu \& Nadri (2010), Mazenc \& NormandCyrot (2013), Hammouri et al. (2006), Nadri et al. (2013), Goffaux et al. (2009), Tellez-Anguiano et al. (2012) and the references therein. These observers offer in some cases fundamental advantages. In particular, we show in the present work that they can be successfully used in cases where neither a static piecewise constant state feedback nor a continuous observer can be applied.

To the best of the authors' knowledge, continuous-discrete observers have never been employed to design stabilizing 
output feedbacks for systems with delay and have never been used in combination with the reduction model approach. In fact, in spite of the fact that delays, sampling, and discrete measurements are frequently encountered simultaneously in control engineering practice, most of the available contributions studied each of these phenomena separately Wang et al. (2014), with the notable exceptions Karafyllis \& Krstic (2012) and Karafyllis \& Krstic 2 (2013), where a nonlinear stabilization result is established for systems with input subject to delay and zero-order hold, the measurements are discrete and with a delay. When particularized to the case of linear time-invariant systems, the main results in Karafyllis \& Krstic (2012), Karafyllis \& Krstic 2 (2013) provide with a feedback whose expressions depend on a sum of past values of the feedback, as those provided by the discretization approach. But it is worth pointing out that they offer the advantage of being insensitive to perturbations in the sampling schedule of the output.

An obstacle to the adaptation of the paper Mazenc \& Normand-Cyrot (2013) to continuous-discrete observers is due to the fact that its main result relies on an operator which needs to be differentiable along the trajectories of the system, but is not along the trajectories of an impulsive system. To overcome this obstacle, we shall use a dynamic extension similar to the one introduced in Mondié \& Michiels (2003).

The observer we shall design converges in the absence of disturbances and is robust with respect to additive disturbances. The system will be stabilized by the control law we shall propose, provided that both the maximum time interval between two consecutive measurements and the largest time interval between two consecutive sampling instants are sufficiently small. We shall prove that, in the case where the output is the entire state, there is convergence of the observer, no matter how large the time intervals between two consecutive measurements are. But the destabilizing effect of the uncertainties on the error equation (i.e. the ultimate bound on the error equation) is proportional to the size of these intervals.

Finally, it is worth observing that the control laws we propose keep the simplicity in their formula of those proposed in Mazenc \& Normand-Cyrot (2013) and that using Wirtinger's inequality (see Liu \& Fridman (2012)), we obtain, through the construction of a continuous-discrete Lyapunov-Krasovskii functional, stabilization conditions in terms of a linear matrix inequality that leads to less restrictive conditions on the size of the sampling intervals than those obtained in Mazenc \& Normand-Cyrot (2013) in the state feedback case.

The paper is organized as follows. An introductory example is given in Section 2. A stabilization result using a continuous-discrete observer is given in Section 3. The result is compared with other control strategies in Section 4. Concluding remarks in Section 5 end the contribution.

Notation, definitions. The notation will be simplified whenever no confusion arise from the context. By $|\cdot|$, we denote the Euclidean norm of vectors of any dimension and the induced norm of matrices of any dimensions. Any $k \times n$ matrix, whose entries are all 0 is denoted $0 . I$ denotes the identity matrix in $\mathbb{R}^{n \times n}$, where $n$ is an arbitrary integer. We adopt the following convention: if $i, j$ are two integers such that $i<j$, then $\{j, \ldots, i\}=\varnothing$ and if $r, s$ are two real numbers such that $r<s$ then $[s, r]=\varnothing$. Let $\omega_{1}>0, \omega_{2}>$ be two constants and the sequence $t_{i}$ be such that $t_{0}=0, t_{i+1}-t_{i} \in\left[\omega_{1}, \omega_{2}\right], \forall i \in \mathbb{N}$. Let $f:[0,+\infty) \rightarrow \mathbb{R}^{l}$ be a function that is continuous over each interval $\left[t_{i}, t_{i+1}\right)$ and such that $\lim _{\substack{t \rightarrow t_{i} \\ t<t_{i}}} f(t)$ exists. Then, for all integer $k \in \mathbb{N}$, we let $f\left(t_{k}^{-}\right)=\lim _{\substack{t \rightarrow t_{k} \\ t<t_{k}}} f(t)$. The notation $P>0$, for $P \in \mathbb{R}^{n \times n}$ means that $P$ is symmetric and positive definite. In symmetric block matrices we use $*$ for terms that are induced by the symmetry.

\section{Motivating example and preliminaries}

\subsection{Motivating example}

In this section, we present an example to illustrate the obstacles to the asymptotic stabilization of systems through feedback in the case where only discrete measurements are available. In Section 3, we will show how these obstacles can be overcome by employing continuous-discrete observers. Consider the two-dimensional system

$$
\left\{\begin{array}{l}
\dot{x}_{1}(t)=x_{2}(t)+\varphi_{1}(t) \\
\dot{x}_{2}(t)=-x_{1}(t)+u(t-h)+\varphi_{2}(t),
\end{array}\right.
$$

with the state $x=\left(x_{1}, x_{2}\right) \in \mathbb{R}^{2}$, the nonnegative constant delay $h$, the output

$$
y(t)=x\left(s_{i}\right)+\epsilon_{i} \in \mathbb{R}^{2} \quad \text { for all } t \in\left[s_{i}, s_{i+1}\right),
$$

the sequence $s_{i}$ defined by $s_{0}=0, s_{i+1}=s_{i}+2 \pi, i \in \mathbb{N}$, the sequence $\epsilon_{i}=\left(\epsilon_{1, i}, \epsilon_{2, i}\right) \in \mathbb{R}^{2}$ which represents errors of measurements, the function $\varphi=\left(\varphi_{1}, \varphi_{2}\right)$ which represents disturbances.

Stabilization without observer. Even when $h=0$, $\varphi_{1}(t)=\varphi_{2}(t)$ for all $t \geq 0$ and $\epsilon_{i}=0$ for all $i \in \mathbb{N}$, no feedback of the type $u(t)=f(y(t))$, where $f$ is a continuous function, stabilizes the system (1) asymptotically. This result is proved in Mazenc \& Fridman (2014)

Classical continuous observer. In Mazenc \& Fridman (2014), we established that, for some arbitrarily small disturbances, classical continuous candidate observers admit solutions which diverge from those of (1). We considered the system defined, for all $i \in \mathbb{N}$ and for all $t \in\left[s_{i}, s_{i+1}\right)$, by

$$
\left\{\begin{aligned}
\dot{\bar{x}}_{1}(t)= & \bar{x}_{2}(t)+k_{1}\left[x_{1}\left(s_{i}\right)+\epsilon_{1, i}-\bar{x}_{1}\left(s_{i}\right)\right] \\
& +k_{2}\left[x_{2}\left(s_{i}\right)+\epsilon_{2, i}-\bar{x}_{2}\left(s_{i}\right)\right] \\
\dot{\bar{x}}_{2}(t)= & -\bar{x}_{1}(t)+u\left(g_{1}(t)-h\right) \\
& +k_{3}\left[x_{1}\left(s_{i}\right)+\epsilon_{1, i}-\bar{x}_{1}\left(s_{i}\right)\right] \\
& +k_{4}\left[x_{2}\left(s_{i}\right)+\epsilon_{2, i}-\bar{x}_{2}\left(s_{i}\right)\right]
\end{aligned}\right.
$$

where the $k_{j}$ 's, $j=1$ to 4 are arbitrary constants and proved that $\lim _{i \rightarrow+\infty}\left|x\left(s_{i}\right)-\bar{x}\left(s_{i}\right)\right|=+\infty$. Thus, even if the system (3) was an observer for the system (1), it would possess very poor robustness properties. However, in the ideal case, for all $i \in \mathbb{N}, \epsilon_{i}=0, \varphi$ is not present and one can initialize (3) with $\bar{x}(0)=y(0)=x(0)$ so that $\bar{x}(t)=x(t)$ 
for all $t \geq 0$ which implies that the system can be asymptotically stabilized with a feedback using $\bar{x}(t)$. However, in general $\epsilon_{0} \neq 0$ and the disturbances $\varphi_{1}$ and $\varphi_{2}$ are acting and then the solutions of (3) may diverge from those of (1). It follows that it cannot be used to asymptotically stabilize the system (1) or to stabilize it in the Input to State Stability sense (for details about the ISS property, see for instance Angeli et al. (2003), Sontag (2001)).

\subsection{Preliminary result}

In this section, we present a technical result. We introduce two constants $\underline{\lambda}, \bar{\lambda}$ satisfying $0<\underline{\lambda}<\bar{\lambda}$ and a sequence of real numbers $\lambda_{i}$ such that for all $i \in \mathbb{N}$,

$$
\lambda_{i} \in[\underline{\lambda}, \bar{\lambda}] \text {. }
$$

We define a sequence of positive numbers $t_{i}$ by

$$
t_{i+1}=t_{i}+\lambda_{i}
$$

for all integer $i \geq 1$ and $t_{0}=0$. To simplify the notation, we also introduce a piecewise constant function:

$$
g_{0}(t)=t_{i}, \forall t \in\left[t_{i}, t_{i+1}\right) .
$$

Next, we consider the impulsive system defined, for all $i \in$ $\mathbb{N}$, by

$$
\begin{gathered}
\dot{z}(t)=\mathcal{A} z(t)+\varphi(t), \forall t \in\left(t_{i}, t_{i+1}\right), \\
z\left(t_{i}\right)=\mathcal{M}_{i} z\left(t_{i}^{-}\right)+\varepsilon\left(g_{0}\left(t_{i}\right)\right),
\end{gathered}
$$

with $z \in \mathbb{R}^{n}, \mathcal{A} \in \mathbb{R}^{n \times n}, \mathcal{M}_{i} \in \mathbb{R}^{n \times n}$ and where $\varepsilon$ : $[0,+\infty) \rightarrow \mathbb{R}^{n}$ and $\varphi:[0,+\infty) \rightarrow \mathbb{R}^{n}$ are continuous functions. We introduce a classical assumption:

Assumption A. There exists a constant $\overline{\mathcal{M}}>0$ such that

$$
\left|\mathcal{M}_{i}\right| \leq \overline{\mathcal{M}}, \forall i \in \mathbb{N} \text {. }
$$

There exists a symmetric positive definite matrix $\mathcal{S} \in \mathbb{R}^{n \times n}$ such that the matrix inequality

$$
\left[\mathcal{M}_{i+1} e^{\mathcal{A} \lambda_{i}}\right]^{\top} \mathcal{S} \mathcal{M}_{i+1} e^{\mathcal{A} \lambda_{i}}-\mathcal{S} \leq-I
$$

is satisfied for all $i \in \mathbb{N}$.

We establish this natural result:

Lemma 1 Let the system (7)-(8) satisfy Assumption A. Then there are constants $\zeta_{j}>0, j=1,2$, such that, for all real numbers $t^{a}, t^{b}, t^{b} \geq t^{a}$, the inequality

$$
\begin{aligned}
\left|z\left(t^{b}\right)\right| \leq & \zeta_{1} e^{-\frac{\ln (2)}{2 \bar{\lambda}}\left(t^{b}-t^{a}\right)}\left|z\left(t^{a}\right)\right| \\
& +\zeta_{2} \sup _{l \in\left[t^{a}, t^{b}\right]}\left\{\left|\varepsilon\left(g_{0}(l)\right)\right|+|\varphi(l)|\right\}
\end{aligned}
$$

holds.

\section{Proof. See Appendix A.}

Remark 1. In accordance with the intuition, the smaller is $\bar{\lambda}$ the larger is the rate of convergence. Moreover, from the proof one can see that the constants $\zeta_{1}$ and $\zeta_{2}$ in general increase when $\bar{\lambda}$ increases.

\section{Main results}

This section is dedicated to the problem of adapting the reduction model approach to the case of linear time-invariant systems whose input is affected by sampling and a constant pointwise delay and for which only some components of the state variable are measured at discrete time instants.

\subsection{System under study}

Let us present the system we study. To begin with, we define two sequences of real numbers $t_{i}$ and $s_{i}$, supposed to be known, as follows: for all $i \in \mathbb{N}$,

$$
t_{0}=0, t_{i+1}=t_{i}+\mu_{i},
$$

with $\mu_{i} \in[\mu, \bar{\mu}]$, where $\mu$ and $\bar{\mu}>0$ are constants and

$$
s_{0}=0, s_{i+1}=s_{i}+\nu_{i},
$$

with $\nu_{i} \in[\underline{\nu}, \bar{\nu}]$, where $\bar{\nu}>0$ and $\underline{\nu}>0$ are constants. To simplify the notation, we define two piecewise constant functions:

$$
\begin{aligned}
& g_{1}(t)=t_{i}, \forall t \in\left[t_{i}, t_{i+1}\right), \\
& g_{2}(t)=s_{i}, \forall t \in\left[s_{i}, s_{i+1}\right) .
\end{aligned}
$$

The system we consider is defined as:

$$
\left\{\begin{array}{l}
\dot{x}(t)=A x(t)+B u\left(g_{1}(t)-h\right)+\varphi(t) \\
y(t)=C x\left(g_{2}(t)\right)+\epsilon\left(g_{2}(t)\right),
\end{array}\right.
$$

where $x \in \mathbb{R}^{n}, A \in \mathbb{R}^{n \times n}, B \in \mathbb{R}^{n \times p}, C \in \mathbb{R}^{q \times n}, u \in \mathbb{R}^{p}$ is the input, $y \in \mathbb{R}^{q}$ is the output, $h \geq 0$ is a constant pointwise delay and $\varphi(t)$ and $\epsilon\left(g_{2}(t)\right)$ are unknown disturbances affecting respectively the plant and the measurements. Both $\varphi$ and $\epsilon$ are supposed to be continuous.

We introduce two assumptions:

Assumption 1. There exist a positive definite matrix $S \in$ $\mathbb{R}^{n \times n}$ and a matrix $L \in \mathbb{R}^{n \times q}$ such that the linear matrix inequality

$$
\left[M_{i+1} e^{A \nu_{i}}\right]^{\top} S M_{i+1} e^{A \nu_{i}}-S \leq-I
$$

with

$$
M_{i}=I+\nu_{i} L C
$$

is satisfied for all $i \in \mathbb{N}$.

Remark 2. (i) When, for all $i \in \mathbb{N}, \nu_{i}=\bar{\nu}$ then Assumption 1 is equivalent to the existence of a matrix $L^{*} \in \mathbb{R}^{n \times q}$ such that $e^{A \bar{\nu}}+L^{*} C$ is Schur stable. (ii) When the pair $(A, C)$ is detectable and for all $i \in \mathbb{N}, \nu_{i}=\bar{\nu}$, one can determine a constant $\bar{\nu}_{*}>0$ such that for all $\bar{\nu} \in\left(0, \bar{\nu}_{*}\right)$, Assumption 1 is satisfied. This fact can be established by observing that the first order approximation with respect to $\bar{\nu}$ of $M_{i+1} e^{A \bar{\nu}}$ is the matrix $I+\bar{\nu}(A+L C)$, which is Schur stable if $L$ is so that the matrix $A+L C$ is Hurwitz and $\bar{\nu}$ is small enough.

Remark 3. Instead of using the dynamic extension involving the variable $\beta$ that is introduced in the theorem below, we could try to apply the reduction model approach with the following auxiliary variable:

$$
\begin{aligned}
z(t) & =e^{A h} \hat{x}(t)+\int_{t-h}^{t} e^{A(t-m)} B K z(m) d m \\
& =e^{A h} x(t)+\int_{t-h}^{t} e^{A(t-m)} B K z(m) d m-e^{A h} \tilde{x}(t),
\end{aligned}
$$


which is similar to the one that was introduced in Mazenc \& Normand-Cyrot (2013). But when one applies this strategy, then one needs to differentiate $z(t)$, which leads to an impulsive system that cannot be easily analyzed. This obstacle leads us to use a dynamic extension of the type of the one introduced in Mondié \& Michiels (2003).

Assumption 2. There exist constant matrices $A_{f} \in$ $\mathbb{R}^{p \times p}, \quad B_{f} \in \mathbb{R}^{p \times n}$, and a positive definite matrix $P \in \mathbb{R}^{(n+p) \times(n+p)}$ such that the linear matrix inequality

$$
G^{\top} P+P G \triangleq-Q<0,
$$

with

$$
G=\left[\begin{array}{cc}
A & B \\
B_{f} & A_{f}
\end{array}\right] \in \mathbb{R}^{(n+p) \times(n+p)}
$$

is satisfied.

Remark 4. When the pair $(A, B)$ is stabilizable, Assumption 2 is satisfied. This fact can be proved by applying the celebrated backstepping technique (for another type of constructions of $A_{f}$ and $B_{f}$, see Mondié \& Michiels (2003)). Let us define two matrices

$$
B_{1}=\left[\begin{array}{c}
0 \\
-B_{f} e^{A h}
\end{array}\right] \text { and } B_{2}=\left[\begin{array}{cc}
0 & e^{A h} B \\
0 & 0
\end{array}\right] .
$$

Assumption 3. The constant $\bar{\mu}$ is such that for some matrix $W=W^{\top}$ the following linear matrix inequality holds:

$$
\left[\begin{array}{lll}
-Q & P B_{2} & \bar{\mu} G^{\top} W \\
* & -\frac{\pi^{2}}{4} W & \bar{\mu} B_{2}^{\top} W \\
* & * & -W
\end{array}\right]<0
$$

Remark 5. Note that there always exists $W>0$ that satisfies the inequality

$$
\left[\begin{array}{ll}
-Q & P B_{2} \\
* & -\frac{\pi^{2}}{4} W
\end{array}\right]<0
$$

Then by Schur complement the LMI (22) holds with a small enough value of $\bar{\mu}$.

\subsection{Continuous-discrete observer}

In this section, we assume that the system (15) satisfies Assumptions 1 and 2 and show that the error equation between the continuous-discrete observer defined, for all $i \in \mathbb{N}$, by

$$
\left\{\begin{array}{l}
\dot{\hat{x}}(t)=A \hat{x}(t)+B u\left(g_{1}(t)-h\right), \forall t \in\left(s_{i}, s_{i+1}\right) \\
\hat{x}\left(s_{i}\right)=\hat{x}\left(s_{i}^{-}\right)-\nu_{i} L\left[y\left(s_{i}\right)-C \hat{x}\left(s_{i}^{-}\right)\right]
\end{array}\right.
$$

where $\hat{x} \in \mathbb{R}^{n}$ and the system (15) is Input to State Stable with respect to $\left(\varphi(t), \epsilon\left(g_{2}(t)\right)\right)$. We introduce the error variable:

$$
\tilde{x}(t)=x(t)-\hat{x}(t)
$$

From (15) and (23), it follows that, for all $i \in \mathbb{N}$ and $t \in$ $\left(s_{i}, s_{i+1}\right)$,

$$
\left\{\begin{aligned}
\dot{\tilde{x}}(t) & =A \tilde{x}(t)+\varphi(t) \\
\tilde{x}\left(s_{i}\right) & =\tilde{x}\left(s_{i}^{-}\right)+\nu_{i} L C \tilde{x}\left(s_{i}^{-}\right)+\epsilon\left(s_{i}\right) \\
& =M_{i} \tilde{x}\left(s_{i}^{-}\right)+\epsilon\left(g_{2}(t)\right)
\end{aligned}\right.
$$

Since Assumption 1 is satisfied, Lemma 1 applies and ensures that there are constants $\eta_{p}>0, p=1,2$ such that the solutions of (25) satisfy, for all real numbers $s$ and $t \geq s \geq 0$ the inequality

$$
|\tilde{x}(t)| \leq \eta_{1} e^{-\frac{\ln (2)}{2 \bar{\nu}}(t-s)}|\tilde{x}(s)|+\eta_{2} \sup _{l \in[s, t]}\left\{|\varphi(l)|+\left|\varepsilon\left(g_{2}(l)\right)\right|\right\} .
$$

Constants $\eta_{p}$ can be found from the proof of Lemma 1.

\subsection{Input-to-State-Stabilization by dynamic output- feedback}

We are ready to state the main result of the paper.

Theorem 1 Let the system (15) satisfy Assumptions 1 to 3. Then the dynamic output feedback defined, for all $i \in \mathbb{N}$, by

$$
\left\{\begin{aligned}
u(t) & =\beta(t) \\
\dot{\beta}(t) & =A_{f} \beta(t) \\
& +B_{f}\left[e^{A h} \hat{x}(t)+\int_{t-h}^{t} e^{A(t-m)} B \beta(m) d m\right] \\
\dot{\hat{x}}(t) & =A \hat{x}(t)+B u\left(g_{1}(t)-h\right), \forall t \in\left(s_{i}, s_{i+1}\right) \\
\hat{x}\left(s_{i}\right) & =\hat{x}\left(s_{i}^{-}\right)-\nu_{i} L\left[y\left(s_{i}\right)-C \hat{x}\left(s_{i}^{-}\right)\right]
\end{aligned}\right.
$$

leads to a closed-loop impulsive system (15), (27), which rewrites as,

$$
\left\{\begin{aligned}
\dot{x}(t) & =A x(t)+B \beta\left(g_{1}(t)-h\right)+\varphi(t) \\
\dot{\beta}(t) & =A_{f} \beta(t) \\
& +B_{f}\left[e^{A h} \hat{x}(t)+\int_{t-h}^{t} e^{A(t-m)} B \beta(m) d m\right] \\
\dot{\hat{x}}(t) & =A \hat{x}(t)+B \beta\left(g_{1}(t)-h\right), \forall t \in\left(s_{i}, s_{i+1}\right) \\
\hat{x}\left(s_{i}\right) & =\hat{x}\left(s_{i}^{-}\right)-\nu_{i} L\left[C x\left(s_{i}\right)-C \hat{x}\left(s_{i}^{-}\right)+\epsilon\left(s_{i}\right)\right],
\end{aligned}\right.
$$

which is such that the inequality

$$
\begin{aligned}
|\varrho(t)| \leq & \kappa_{1}\left[|x(s)|+|\hat{x}(s)|+\sup _{l \in[s-h, s]}|\beta(l)|\right] e^{\kappa_{3}(s-t)} \\
& \left.+\kappa_{2} \sup _{l \in[s, t]}\{\mid \varphi(l))|+| \epsilon\left(g_{2}(l)\right) \mid\right\}
\end{aligned}
$$

with $\varrho(t)=(x(t), \beta(t), \hat{x}(t))$, is satisfied for some constants $\kappa_{p}>0, p=1,2,3$, for all real numbers $t \geq s \geq 0$.

Proof of Theorem 1: see Appendix B.

Remark 6. It is worth mentioning that in Theorem 1, no constraint on the size of the delay $h$ is imposed.

Remark 7. The example (1) also illustrates how the technique we propose may be useful to lazy samplers, i.e. in the case where only a low number of sampling instants is desired. Indeed, the system (1) satisfies Assumption 2 because it is controllable and it satisfies Assumption 1 because $L=-\frac{1}{2 \pi} I$ gives $M_{i}=0$ for all $i \in \mathbb{N}$. Therefore Theorem 1 applies and provides with exponentially stabilizing control laws for (1) for arbitrarily large measurement sampling intervals.

Remark 8. By induction, one can prove that, for any initial condition $x(0), \hat{x}(0)$ and $\beta(l), l \in[-h, 0]$, where $\beta$ is continuous function, there is a unique absolutely continuous solution of the system (28) on any interval $\left[s_{i}, s_{i+1}\right)$.

Remark 9. From Remark 1 and the proof of Theorem 1 , it appears that the size of $\bar{\nu}$ has an influence on the ISS inequality (29). Roughly speaking, the smaller is $\bar{\nu}$, the smaller is the right-hand-side of (29). We also wish to point 
out that the proof of Theorem 1 is constructive: the values of the constants $\kappa_{i}$ can always be determined.

Remark 10. Theorem 1 is a result for a system with additive disturbances only. It may be worth considering other types of uncertainties. In particular, by borrowing ideas from the contribution Chen et al. (2014), we conjecture that we can extend Theorem 1 to the case, important from an applied point of view, where there are uncertainties on the matrices $A$ and $C$.

\section{Comparison with other stabilization techniques}

In this section, we show that Theorem 1 applies in cases where other classical techniques do not.

\subsection{Stabilization through state feedback}

Now we show that Theorem 1 can be used to determine asymptotically stabilizing control laws in cases where the output is given by $y(t)=x\left(s_{i}\right)$, for all integer $i$ and $t \in$ $\left[s_{i}, s_{i+1}\right)$ and no time invariant static feedback renders the origin of system (15) asymptotically stable. For the sake of simplicity, consider the system (15) with $h=0, g_{1}(t)=t$, and for all $i \in \mathbb{N}, s_{i+1}-s_{i}=\bar{\nu}>0$. Suppose in addition that it satisfies Assumption 2 i.e. that the pair $(A, B)$ is stabilizable. Hence, the system is defined for all $i \in \mathbb{N}$ by

$$
\left\{\begin{array}{l}
\dot{x}(t)=A x(t)+B u(t) \\
y(t)=x\left(s_{i}\right)+\epsilon\left(s_{i}\right)
\end{array}, \quad \forall t \in\left[s_{i}, s_{i+1}\right) .\right.
$$

It is well-known that, in general, there are real numbers $\bar{\nu}>0$ such that there does not exist a constant matrix $F$ such that the system (30) is stabilized by the control

$$
u(t)=F x\left(s_{i}\right) \quad, \quad \forall t \in\left[s_{i}, s_{i+1}\right),
$$

even if $\epsilon$ is identically equal to zero. We have seen in Section 2 through an example that in some cases there does not even exist a nonlinear feedback defined as $u(t)=f\left(x\left(s_{i}\right)\right)$ for all $t \in\left[s_{i}, s_{i+1}\right)$ that asymptotically stabilizes this system in the absence of $\epsilon$.

Now, observe that, with the notation of Section 3.1, $C=I$ and $\nu_{i}=\bar{\nu}$ for all $i \in \mathbb{N}$, so that the choice $L=-\frac{1}{\bar{\nu}} I$ gives $M_{i}=0$ for all $i \in \mathbb{N}$. Therefore Assumption 1 is satisfied with $S=I$. Then for any $\bar{\nu}>0$, Theorem 1 provides with control laws rendering the system (30) Input to State Stable with respect to the uncertainty $\epsilon\left(g_{2}(t)\right)$. Notice that in this particular case, the dynamic output feedback is defined, for all $i \in \mathbb{N}$, by

$$
\left\{\begin{aligned}
u(t) & =\beta(t) \\
\dot{\beta}(t) & =A_{f} \beta(t) \\
& +B_{f}\left[e^{A h} \hat{x}(t)+\int_{t-h}^{t} e^{A(t-m)} B \beta(m) d m\right] \\
\dot{\hat{x}}(t) & =A \hat{x}(t)+B u\left(g_{1}(t)-h\right), \forall t \in\left(s_{i}, s_{i+1}\right) \\
\hat{x}\left(s_{i}\right) & =y\left(s_{i}\right)=x\left(s_{i}\right)+\epsilon\left(s_{i}\right),
\end{aligned}\right.
$$

for suitably chosen matrices $A_{f}$ and $B_{f}$.

Example. Consider the system (15), where $n=2$ and

$$
A=\left[\begin{array}{cc}
0 & 1 \\
0 & -0.1
\end{array}\right], \quad B=\left[\begin{array}{c}
0 \\
0.1
\end{array}\right], \quad C=I .
$$

The stability analysis of the unperturbed closed-loop system under the controller

$$
u\left(g_{1}(t)-h\right)=F x\left(t_{k}-h\right), F=-\left[\begin{array}{ll}
3.75 & 11.5
\end{array}\right],
$$

for all $t_{k} \leq t<t_{k+1}$, with $h \geq 0$ and $t_{k+1}-t_{k} \leq \bar{\nu}$ has been studied e.g. in Liu \& Fridman (2012). Note that the system with the continuous control $u(t)=F x(t-h)$ is input-to-state stable for $h \leq 1.16$ and becomes unstable for $h>1.17$ (see p. 308 of Fridman (2014)). This means that the existing methods for systems with a delay belonging to the interval $[h, h+\bar{\mu}$ ) (see Liu \& Fridman (2012) and the references therein) cannot guarantee the stability of the closed-loop sampled-data system if $h>1.17$ (even for small $\bar{\mu})$.

We consider next the dynamic output-feedback (32) and choose $A_{f}=F A+F$ and $B_{f}=F B-I$, where $A$ and $B$ are the matrices in (33) (we designed $A_{f}$ and $B_{f}$ by applying the backstepping approach). Note that this choice leads to $e i g(G)=e i g(A+B F) \bigcup e i g(-I)$. Thus, the eigenvalues of $G$ are $-0.5,-0.75$ and -1 . As mentioned above, for the case of continuous-time control with $g_{1}(t)=t$, the feedback (32) leads to input-to-state stability with respect to $\left(\varphi, \epsilon\left(g_{2}\right)\right)$ for all $h>0$ and $\bar{\nu}>0$.

Consider further the case of the zero-order hold with $g_{1}(t)=t_{i}$. By verifying the feasibility of the LMI (22) for the values of $h$ given in Table 4.1, we find the resulting maximum values of $\bar{\mu}$ that preserve input-to-state-stability. As expected, by the predictor-based control we are able to stabilize systems for arbitrary large values of $h$ (and not only for small enough $h$ as in the existing sampleddata literature). Since $A$ is not Hurwitz and, after a small threshold, $\left|B_{2}\right|$ (cf. (21)) grows for growing $h$, the resulting values of $\bar{\mu}$ become smaller for larger $h$. Therefore, in case of the zero-order hold, our approach allows arbitrary large measurement sampling intervals (bounded by $\bar{\nu}>0$ ), whereas it requires fast enough updating times of the zero-order hold bounded by $\bar{\mu}$. This is an advantage over the predictor-based approach of Mazenc \& Normand-Cyrot (2013), where the measurements of the state are supposed to be continuous, whereas updating intervals are small enough.

\begin{tabular}{|c||c|c|c|c|c|}
\hline $\bar{\mu}$ & 2 & 5 & 10 & 20 & 100 \\
\hline$h$ & 0.91 & 0.83 & 0.77 & 0.72 & 0.69 \\
\hline
\end{tabular}

Max. value of $\bar{\mu}$ for different $h$

\subsection{Continuous observers}

The following type of continuous observer:

$$
\dot{\bar{x}}(t)=A \bar{x}(t)+B u\left(g_{1}(t)-h\right)+L\left[y\left(s_{i}\right)-C \bar{x}\left(s_{i}\right)\right],
$$

for all $t \in\left[s_{i}, s_{i+1}\right)$ and $i \in \mathbb{N}$, where $\bar{x} \in \mathbb{R}^{n}$ and $L \in \mathbb{R}^{n \times q}$ is a constant matrix, is classically used when the system with discrete measurements (15) is considered Fridman (2014), Seuret et al. (2006). One can check readily that an observer of this type can be easily combined with the reduction model approach, and in particular with the result of 
Mazenc \& Normand-Cyrot (2013). However, as illustrated in Section 2, continuous-discrete observers converge to (15) in cases where none of the observers of the type (35) does.

\section{Conclusion}

We solved an observer-based output feedback stabilization problem for linear time-invariant systems with a pointwise arbitrarily large constant delay and zero-order-hold input and discrete measurements. The proposed technique relies on the introduction of a continuous-discrete observer and the adaptation of the celebrated reduction model approach. Much remains to be done. Other types of delays and timevarying systems may be considered. Nonlinear extensions borrowing ideas from Karafyllis \& Krstic (2012), Nadri et al. (2004) may be the subject of further studies.

\section{References}

V. Andrieu, M. Nadri, Observer design for Lipschitz systems with discrete-time measurements. 49th IEEE Conference on Decision and Control, Atlanta, USA, pp. $6522-$ $6527,2010$.

D. Angeli, E. D. Sontag, Y. Wang, Input-to-state stability with respect to inputs and their derivatives. Internat. J. Robust Nonlinear Control, 13 (11), pp.1035-1056, 2003.

Z. Artstein, Linear systems with delayed controls: A reduction. IEEE Trans. on Automatic Control, Vol. 27, No. 4, pp. 869-879, 1982.

B. Castillo-Toledo, S. Di Gennaro, G. Sandoval Castro, Stability Analysis for a Class of Sampled Nonlinear Systems with Time-Delay. 49th IEEE Conference on Decision and Control, Atlanta, USA, pp.1575-1580, 2010.

W.-H. Chen, W. Yang, X. Lu, Impulsive observer-based stabilisation of uncertain linear systems. Control Theory Applications, IET, Issue: 3, (8), pp. 149-159, 2014.

F. Deza, E. Busvelle, J.P. Gauthier, D. Rakotopara, High gain estimation for nonlinear systems. Systems \& Control Letters, 18 (4), pp. 295-299, 1992.

E. Fridman, A refined input delay approach to sampled-data control. Automatica, Vol. 46, pp. 421-427, 2010.

E. Fridman, Introduction to Time-Delay Systems. Series: Analysis and Control Series: Systems \& Control: Foundations \& Applications, Birkhauser Boston, 2014.

G. Goffaux, A. Vande Wouwer, O. Bernard, Improving continuous-discrete interval observers with application to microalgae-based bioprocess. Journal of Process Control, 19 (7), pp. 1182-1190, 2009.

A. Gonzalez, P. Garcia, P. Albertos, P. Castillo, R. Lozano, Robustness of a discrete-time predictor-based controller for time-varying measurement delay. Control Engineering Practice 20, pp. 102-110, 2012.

H. Hammouri, N. Nadri, R. Mota, Constant gain observer design for continuous-discrete time uniformly observable systems. 45th IEEE Conference on Decision and Control, San Diego, CA, USA, pp. 5406-5411, 2006.

A. H. Jazwinski, Stochastic processes and filtering theory. Mathematics in Science and Engineering, 1970.

I. Karafyllis, M. Krstic, Nonlinear Stabilization under Sam- pled and Delayed Measurements, and with Inputs Subject to Delay and Zero-Order Hold. IEEE Trans. on Automatic Control, Vol. 57, pp. 1141-1154, 2012.

I. Karafyllis, M. Krstic, Stabilization of Nonlinear Delay Systems Using Approximate Predictors and High-Gain Observers. Automatica, Vol. 49, pp. 3623-3631, 2013.

N. Kazantzis, K. T. Chong, A.G. Parlos, Control-Relevant Discretization of Nonlinear Systems With Time-Delay Using Taylor-Lie Series. ASME Journal of Dynamic Systems, Measurement, and Control Vol. 127/153, pp. 153159, March 2005.

K. Liu, E. Fridman. Wirtinger's Inequality and LyapunovBased Sampled-Data Stabilization. Automatica, Vol. 48, pp. 102-108, 2012.

D.Q. Mayne, Control of linear systems with time delay. Electronics Letters, Vol. 4. no. 20, 4th October 1968.

F. Mazenc, E. Fridman, Predictor-based sampled-data stabilization via continuous-discrete observers. 53th IEEE Conference on Decision and Control, pp. 1614-1619, Los Angeles, USA, 2014.

F. Mazenc, M. Malisoff, Local Stabilization of Nonlinear Systems through the Reduction Model Approach. IEEE Trans. on Automatic Control, Vol. 59, No. 8, pp. 20682081, 2014.

F. Mazenc, M. Malisoff, S.I. Niculescu, Reduction Model Approach for Linear Time-Varying Systems with Delays. IEEE Trans. on Automatic Control, Vol. 59, No. 8, pp. 2068-2081, 2014.

F. Mazenc, S.-I. Niculescu, M. Krstic, Lyapunov-Krasovskii Functionals and Application to Input Delay Compensation for Linear Time-Invariant Systems. Automatica, Vol. 48, Issue 7, pp. 1317-1323, July 2012.

F. Mazenc, D. Normand-Cyrot, Reduction Model Approach for Linear Systems With Sampled Delayed Inputs. IEEE Trans. on Automatic Control, Vol. 58, Issue 5, pp. 12631268, 2013.

S. Mondié, W. Michiels, Finite Spectrum Assignment of Unstable Time-Delay System With a Safe Implementation. IEEE Trans. on Automatic Control, Vol. 48, No. 12, pp. 2207-2212, Dec. 2003.

M. Nadri, H. Hammouri, C. Astorga, Observer Design for Continuous-Discrete Time State Affine Systems up to Output Injection. European Journal of Control, Vol. 10, pp. 252-263, 2004.

M. Nadri, H. Hammouri, R. Mota Grajales, Observer Design for Uniformly Observable Systems With Sampled Measurements. IEEE Trans. on Automatic Control, Vol. 58, No. 3 pp. 757-762, March 2013.

A. Seuret, F. Michaut, J. Richard, T. Divoux, Networked control using GPS synchronization. 2006 American Control Conference, pp. 4195-4200, New York, USA.

E. Sontag, The ISS philosophy as a unifying framework for stability-like behavior. In Nonlinear control in the year 2000, Vol. 2 (Paris), volume 259 of Lecture Notes in Control and Inform. Sci., pp. 443-467. Springer, London, 2001.

A. C. Tellez-Anguiano, C. M. Astorga-Zaragoza, E. Alcorta-Garcia, B. Targui, E. Quintero-Marmol, M. Adam-Medina, V. H. Olivares-Peregrino, Nonlinear 
Continuous-Discrete Observer Application to Distillation Columns. International Journal of Innovative Computing, Information and Control. Vol. 8, No. 1(B), January 2012.

H. P. Wang, Y. Tian, N. Christov, Piecewise-continuous observers for linear systems with sampled and delayed output. International Journal of Systems Science, 2014.

E. Witrant, C. Canudas-de-Wit, D. Georges, M. Alamir, Remote Stabilization via Communication Networks with a Distributed Control Law. IEEE Trans. on Automatic Control, Vol. 52, pp. 1480-1485, Aug. 2007.

\section{A Proof of Lemma 1}

Let $i \in \mathbb{N}$. By integrating (7), we obtain

$$
z\left(t_{i+1}^{-}\right)=e^{\mathcal{A} \lambda_{i}} z\left(t_{i}\right)+\int_{t_{i}}^{t_{i+1}} e^{\left(t_{i+1}-\ell\right) A} \varphi(\ell) d \ell .
$$

Then (8) gives

$$
r_{i+1}=\mathcal{M}_{i+1} e^{\mathcal{A} \lambda_{i}} r_{i}+\varpi_{i}
$$

with the simplifying notations $r_{i}=z\left(t_{i}\right)$

$$
\varpi_{i}=\varepsilon\left(g_{0}\left(t_{i+1}\right)\right)+\int_{t_{i}}^{t_{i+1}} \mathcal{M}_{i+1} e^{\left(t_{i+1}-\ell\right) A} \varphi(\ell) d \ell .
$$

To analyze (A.2), we introduce the candidate Lyapunov function $\mathcal{V}(r)=r^{\top} \mathcal{S} r$. Then

$$
\begin{aligned}
\mathcal{V}\left(r_{i+1}\right)= & r_{i}^{\top}\left(\mathcal{M}_{i+1} e^{\mathcal{A} \lambda_{i}}\right)^{\top} \mathcal{S} \mathcal{M}_{i+1} e^{\mathcal{A} \lambda_{i}} r_{i} \\
& +2\left(\mathcal{M}_{i+1} e^{\mathcal{A} \lambda_{i}} r_{i}\right)^{\top} \mathcal{S} \varpi_{i}+\varpi_{i}^{\top} \mathcal{S} \varpi_{i} .
\end{aligned}
$$

Since $\mathcal{S}$ is symmetric and positive definite, for any $a>0$,

$$
\begin{aligned}
\mathcal{V}\left(r_{i+1}\right) \leq & (1+a) r_{i}^{\top}\left(\mathcal{M}_{i+1} e^{\mathcal{A} \lambda_{i}}\right)^{\top} \mathcal{S} \mathcal{M}_{i+1} e^{\mathcal{A} \lambda_{i}} r_{i} \\
& +\left(1+\frac{4}{a}\right) \varpi_{i}^{\top} \mathcal{S} \varpi_{i} .
\end{aligned}
$$

From Assumption A, we deduce that

$$
\begin{aligned}
\mathcal{V}\left(r_{i+1}\right) & \leq(1+a) r_{i}^{\top}(\mathcal{S}-I) r_{i}+\left(1+\frac{4}{a}\right) \varpi_{i}^{\top} \mathcal{S} \varpi_{i} \\
& \leq(1+a)\left(1-\frac{1}{|\mathcal{S}|}\right) \mathcal{V}\left(r_{i}\right)+\left(1+\frac{4}{a}\right)|\mathcal{S}|\left|\varpi_{i}\right|^{2}
\end{aligned}
$$

From (10), we deduce that $1 \leq|\mathcal{S}|$. Choosing $a$ such that $a(1-|\mathcal{S}|) \leq \frac{1}{2|\mathcal{S}|}$, we obtain

$$
\begin{aligned}
\mathcal{V}\left(r_{i+1}\right) & \leq\left(1-\frac{1}{2|\mathcal{S}|}\right) \mathcal{V}\left(r_{i}\right)+\alpha_{0}\left|\varpi_{i}\right|^{2} \\
& \leq \frac{1}{2} \mathcal{V}\left(r_{i}\right)+\alpha_{0}\left|\varpi_{i}\right|^{2}
\end{aligned}
$$

with $\alpha_{0}=(1+8|\mathcal{S}|(|\mathcal{S}|-1))|\mathcal{S}|$. Then, for all integers $i$ and $j \geq i$, the following inequality holds:

$$
\mathcal{V}\left(r_{j}\right) \leq \frac{1}{2^{j-i}} \mathcal{V}\left(r_{i}\right)+2 \alpha_{0} \sup _{m \in\{i, \ldots, j-1\}}\left\{\left|\varpi_{m}\right|^{2}\right\}
$$

Let $s_{m}$ be the smallest eigenvalue of $\mathcal{S}$. From this inequality, we deduce that, for all integers $i$ and $j \geq i$, the inequality

$$
\left|z\left(t_{j}\right)\right| \leq \alpha_{1} e^{-\frac{\ln (2)}{2}(j-i)}\left|z\left(t_{i}\right)\right|+\alpha_{2} \sup _{m \in\{i, \ldots, j-1\}}\left\{\left|\varpi_{m}\right|\right\}
$$

with $\alpha_{1}=\sqrt{\frac{|\mathcal{S}|}{s_{m}}}$ and $\quad \alpha_{2}=\sqrt{\frac{2 \alpha_{0}}{s_{m}}}$ is satisfied.

Next, let us consider two real numbers $t^{a}<t^{b}$. Then, there exists a unique pair of integers $(j, k)$ such that

$$
t_{j} \leq t^{b}<t_{j+1}, \quad t_{k} \leq t^{a}<t_{k+1} .
$$

Let us distinguish between 2 cases:

First case: $j=k$. Then we deduce from (7) and (4) that

$$
\left|z\left(t^{b}\right)\right| \leq e^{\bar{\lambda}(|\mathcal{A}|+1)-\left(t^{b}-t^{a}\right)}\left|z\left(t^{a}\right)\right|+\alpha_{3} \sup _{\ell \in\left[t^{a}, t^{b}\right]}\{|\varphi(\ell)|\} .
$$

with $\alpha_{3}=e^{\bar{\lambda}|\mathcal{A}|}$.

Second case: $j>k$. Then, necessarily the inequalities $t^{a}<$ $t_{k+1} \leq t_{j} \leq t^{b}$ are satisfied. From (A.9), we deduce that

$$
\left|z\left(t^{b}\right)\right| \leq \alpha_{3}\left|z\left(t_{j}\right)\right|+\alpha_{3} \int_{t_{j}}^{t^{b}}|\varphi(\ell)| d \ell .
$$

From (A.8) we deduce that

$$
\begin{gathered}
\left|z\left(t^{b}\right)\right| \leq \alpha_{3} \alpha_{1} e^{-\frac{\ln (2)}{2}(j-k-1)}\left|z\left(t_{k+1}\right)\right| \\
+\alpha_{2} \alpha_{3} \sup _{m \in\{k+1, \ldots, j-1\}}\left\{\left|\varpi_{m}\right|\right\}+\alpha_{3} \int_{t_{j}}^{t^{b}}|\varphi(\ell)| d \ell .
\end{gathered}
$$

From (8) and (9), it follows that

$$
\left|z\left(t_{k+1}\right)\right| \leq \overline{\mathcal{M}}\left|z\left(t_{k+1}^{-}\right)\right|+\left|\epsilon\left(g_{0}\left(t_{k+1}\right)\right)\right| .
$$

Bearing in mind (A.9), by integrating (7) and using (4), we obtain that

$$
\left|z\left(t_{k+1}^{-}\right)\right| \leq \alpha_{3}\left|z\left(t^{a}\right)\right|+\alpha_{3} \int_{t^{a}}^{t_{k+1}}|\varphi(\ell)| d \ell,
$$

which, in combination with (A.13), implies that

$$
\begin{aligned}
\left|z\left(t_{k+1}\right)\right| \leq & \overline{\mathcal{M}} \alpha_{3}\left|z\left(t^{a}\right)\right|+\left|\epsilon\left(g_{0}\left(t_{k+1}\right)\right)\right| \\
& +\overline{\mathcal{M}} \alpha_{3} \int_{t^{a}}^{t_{k+1}}|\varphi(\ell)| d \ell .
\end{aligned}
$$

This inequality and (A.12) give

$$
\begin{aligned}
\left|z\left(t^{b}\right)\right| \leq & \alpha_{3}^{2} \alpha_{1} e^{-\frac{\ln (2)}{2}(j-k-1) \overline{\mathcal{M}}}\left|z\left(t^{a}\right)\right| \\
& +\alpha_{3} \alpha_{1} e^{-\frac{\ln (2)}{2}(j-k-1)}\left|\epsilon\left(g_{0}\left(t_{k+1}\right)\right)\right| \\
& +\alpha_{3}^{2} \alpha_{1} e^{-\frac{\ln (2)}{2}(j-k-1) \overline{\mathcal{M}} \int_{t_{k+1}}^{t_{k+1}}|\varphi(\ell)| d \ell} \\
& +\alpha_{2} \alpha_{3} \sup _{m \in\{k+1, \ldots, j-1\}}\left\{\left|\varpi_{m}\right|\right\} \\
& +\alpha_{3} \int_{t_{j}}^{t^{b}}|\varphi(\ell)| d \ell .
\end{aligned}
$$

Now, observe that (4) implies that

$$
t^{b}-t^{a} \leq t_{j}-t_{k}+\bar{\lambda} \leq \bar{\lambda}(j-k+1)
$$

From the last inequality and (A.16) we can deduce (11).

\section{B Proof of Theorem 1}

Consider a trajectory $(x(t), \beta(t), \hat{x}(t))$ of the system (15)(27). Denote

$$
r(t)=e^{A h} x(t)+\int_{t-h}^{t} e^{A(t-l)} B \beta(l) d l .
$$

Then, simple calculations give, for all $i \in \mathbb{N}$ and $t \in$ $\left[t_{i}, t_{i+1}\right)$

$$
\left\{\begin{aligned}
\dot{r}(t)= & A r(t)+B \beta(t) \\
& +e^{A h} B\left[\beta\left(t_{i}-h\right)-\beta(t-h)\right]+e^{A h} \varphi(t) \\
\dot{\beta}(t)= & A_{f} \beta(t)+B_{f} r(t)-B_{f} e^{A h} \tilde{x}(t) .
\end{aligned}\right.
$$


Moreover, from the result in Section 3.2, we deduce that $\tilde{x}(t)=x(t)-\hat{x}(t)$ satisfies an inequality of the type (26). Now, let us analyze the Input to State Stability of (B.2) with respect to $(\varphi, \tilde{x})$ regarded as an the input. Denoting $\xi=\left(r^{\top} \beta^{\top}\right)^{\top}$, we rewrite this system as follows, for all $t \in\left[t_{i}, t_{i+1}\right)$,

$$
\begin{aligned}
\dot{\xi}(t)= & G \xi(t)+B_{2}\left[\xi\left(t_{i}-h\right)-\xi(t-h)\right]+B_{1} \tilde{x}(t) \\
& +B_{3} \varphi(t),
\end{aligned}
$$

with $B_{1}$ and $B_{2}$ defined in (21) and

$$
B_{3}=\left[\begin{array}{c}
e^{A h} \\
0
\end{array}\right] \text {. }
$$

Now, consider the following piecewise-continuous in time Lyapunov-Krasovskii functional, define with an abuse of notation, by

$$
\begin{aligned}
& V(t)=\xi(t)^{\top} P \xi(t)+V_{D}(t)-\frac{\pi^{2}}{4} V_{W}(t), \\
& V_{D}(t)=\bar{\mu}^{2} e^{2 \alpha \bar{\mu}} \int_{t_{i}-h}^{t} e^{-2 \alpha(t-s)} \dot{\xi}^{\top}(s) W \dot{\xi}(s) d s \\
& V_{W}(t)=\int_{t_{i}-h}^{t-h}\left[\xi(s)-\xi\left(t_{i}-h\right)\right]^{\top} W\left[\xi(s)-\xi\left(t_{i}-h\right)\right] d s,
\end{aligned}
$$

for all $i \in \mathbb{N}, t \in\left[t_{i}, t_{i+1}\right)$ and where $P$ and $W$ are the matrices given by Assumptions 2 and 3 and $\alpha>0$ is a tuning parameter. Let us mention that the integral terms of $V$ with $\alpha=0$ were introduced in Liu \& Fridman (2012) for the stability analysis of sampled-data systems. Since $\left[\xi(s)-\xi\left(t_{i}-h\right)\right]_{\mid s=t_{i}-h}=0$, by the Wirtinger's inequality (see for instance Liu \& Fridman (2012)), we obtain $V_{D}(t)-$ $\frac{\pi^{2}}{4} V_{W}(t) \geq 0$. Moreover, $V_{D}(t)-\frac{\pi^{2}}{4} V_{W}(t)$ vanishes at $t=$ $t_{i}$. Hence, the condition

$$
V\left(t_{i}^{-}\right) \geq V\left(t_{i}\right)
$$

holds. Then, by using arguments of Fridman (2010) one can prove that, the following condition along the trajectories of (B.2) guarantees its Input to State Stability:

$$
\Omega(t)=\dot{V}(t)+\rho V(t)-\gamma\left(|\tilde{x}(t)|^{2}+|\varphi(t)|^{2}\right) \leq 0,
$$

where $\rho>0, \gamma>0$ are some constants.

Differentiating $V$ along the trajectories of (B.2), we obtain, for all $t \in\left[t_{i}, t_{i+1}\right)$,

$$
\begin{aligned}
& \dot{V}(t)=-2 \alpha V_{D}(t)+\bar{\mu}^{2} e^{2 \alpha \bar{\mu}} \dot{\xi}^{\top}(t) W \dot{\xi}(t) \\
& \quad+2 \xi(t)^{\top} P\left[G \xi(t)+B_{2}\left(\xi\left(t_{i}-h\right)-\xi(t-h)\right)+B_{1} \tilde{x}(t)\right] \\
& \quad-\frac{\pi^{2}}{4}\left[\xi(t-h)-\xi\left(t_{i}-h\right)\right]^{\top} W\left[\xi(t-h)-\xi\left(t_{i}-h\right)\right] \\
& \quad+2 \xi(t)^{\top} P B_{3} \varphi(t) .
\end{aligned}
$$

Next, taking into account Assumption 2, we obtain

$$
\begin{aligned}
\dot{V}(t) \leq & -\xi(t)^{\top} Q \xi(t)-2 \alpha V_{D}(t)+2 \xi(t)^{\top} P B_{2} v(t) \\
& +\bar{\mu}^{2} e^{2 \alpha \bar{\mu}} \dot{\xi}^{\top}(t) W \dot{\xi}(t)-\frac{\pi^{2}}{4} v(t)^{\top} W v(t) \\
& +2 \xi(t)^{\top} P B_{1} \tilde{x}(t)+2 \xi(t)^{\top} P B_{3} \varphi(t),
\end{aligned}
$$

with $v(t)=\xi\left(t_{i}-h\right)-\xi(t-h)$. As an immediate consequence, we have,

$$
\begin{aligned}
\Omega(t) & \leq-\xi(t)^{\top} Q \xi(t)-2 \alpha V_{D}(t)+2 \xi(t)^{\top} P B_{2} v(t) \\
& +\bar{\mu}^{2} e^{2 \alpha \mu} \dot{\xi}^{\top}(t) W \dot{\xi}(t)-\frac{\pi^{2}}{4} v(t)^{\top} W v(t) \\
& +2 \xi(t)^{\top} P B_{1} \tilde{x}(t)+\rho V(t) \\
& -\gamma\left(|\tilde{x}(t)|^{2}+|\varphi(t)|^{2}\right)+2 \xi(t)^{\top} P B_{3} \varphi(t) .
\end{aligned}
$$

Now, observe that $V(t) \leq a \xi(t)^{\top} Q \xi(t)+V_{D}(t)$, with $a=$ $\frac{|P|}{\lambda_{\min }(Q)}$. It follows that

$$
\begin{aligned}
\Omega(t) \leq & (-1+a \rho) \xi(t)^{\top} Q \xi(t)+(\rho-2 \alpha) V_{D}(t) \\
& +2 \xi(t)^{\top} P B_{2} v(t)+\bar{\mu}^{2} e^{2 \alpha \bar{\mu}} \dot{\xi}^{\top}(t) W \dot{\xi}(t) \\
& -\frac{\pi^{2}}{4} v(t)^{\top} W v(t)+2 \xi(t)^{\top} P B_{1} \tilde{x}(t) \\
& -\gamma\left(|\tilde{x}(t)|^{2}+|\varphi(t)|^{2}\right)+2 \xi(t)^{\top} P B_{3} \varphi(t) .
\end{aligned}
$$

Denote $\eta(t)=\left(\xi(t)^{\top} v(t)^{\top} \tilde{x}(t)^{\top}\right)^{\top}$ and let $\rho$ be smaller that $2 \alpha$. Then

$$
\begin{aligned}
\Omega(t) & \leq(-1+a \rho) \xi(t)^{\top} Q \xi(t)+2 \xi(t)^{\top} P B_{2} v(t) \\
& +\bar{\mu}^{2} e^{2 \alpha \bar{\mu}} \dot{\xi}^{\top}(t) W \dot{\xi}(t)-\frac{\pi^{2}}{4} v(t)^{\top} W v(t) \\
& +2 \xi(t)^{\top} P B_{1} \tilde{x}(t)-\gamma|\tilde{x}(t)|^{2}+2 \xi(t)^{\top} P B_{3} \varphi(t) \\
& \leq \eta^{\top}(t) \Xi \eta(t)+\bar{\mu}^{2} e^{2 \alpha \bar{\mu}} \dot{\xi}^{\top}(t) W \dot{\xi}(t) \\
& +2 \xi(t)^{\top} P B_{3} \varphi(t),
\end{aligned}
$$

where

$$
\Xi=\left[\begin{array}{cccc}
-(1-a \rho) Q & P B_{2} & P B_{1} & P B_{3} \\
* & -\frac{\pi^{2}}{4} W & 0 & 0 \\
* & * & -\gamma I & 0 \\
* & * & * & -\gamma I
\end{array}\right]
$$

Finally, using the expression of $\dot{\xi}(t)$ and applying the Schur complements to $\bar{\mu}^{2} e^{2 \alpha \bar{\mu}} \dot{\xi}^{\top}(t) W \dot{\xi}(t)$ in (B.12) we conclude that $\Omega$ is nonnegative if the linear matrix inequality:

$$
\left[\begin{array}{ccccc}
-(1-a \rho) Q & P B_{2} & P B_{1} & P B_{3} & \bar{\mu} e^{\alpha \bar{\mu}} G^{\top} W \\
* & -\frac{\pi^{2}}{4} W & 0 & 0 & \bar{\mu} e^{\alpha \bar{\mu}} B_{2}^{\top} W \\
* & * & -\gamma I & 0 & \bar{\mu} e^{\alpha \bar{\mu}} B_{1}^{\top} W \\
* & * & * & -\gamma I & \bar{\mu} e^{\alpha \bar{\mu}} B_{3}^{\top} W \\
* & * & * & * & -W^{\top}
\end{array}\right]<0
$$

is satisfied. Note that (22) implies that the latter inequality holds for small enough $\rho>0, \alpha>0$ and large enough $\gamma$. Then it follows that there are constants $\varsigma_{p}>0, p=1,2,3$, such that, for all $t \geq s \geq 0$, the inequality

$$
|\xi(t)| \leq \varsigma_{1} e^{-\varsigma_{2}(t-s)}|\xi(s)|+\varsigma_{3} \sup _{l \in[s, t]}\{|\varphi(t)|+|\tilde{x}(l)|\}
$$

is satisfied. This property and (26) imply that there are constants $\varsigma_{p}>0, p=4,5,6$ such that, for all $t \geq s$,

$$
\begin{aligned}
|\xi(t)|+|\tilde{x}(t)| \leq & \varsigma_{4} e^{-\varsigma_{5}(t-s)}(|\xi(s)|+|\tilde{x}(s)|) \\
& +\varsigma_{6} \sup _{l \in[s, t]}\{\psi(l)\}
\end{aligned}
$$

with $\psi(l)=\left|\varepsilon\left(g_{2}(l)\right)\right|+|\varphi(l)|$. From the definition of $r$, the inequality $|r(t)| \leq|\xi(t)|$ and (B.15), we obtain

$$
\begin{aligned}
\bar{x}(t) \leq & e^{h|A|}|r(t)|+e^{h|A|}|B| S_{\beta}(t) \\
\leq & \varsigma_{7} e^{\varsigma_{5}(s-t)}(|\xi(s)|+|\tilde{x}(s)|) \\
& +\varsigma_{8} \sup _{l \in[s, t]}\{\psi(l)\}+\varsigma_{9} S_{\beta}(t)
\end{aligned}
$$


with $\varsigma_{7}=e^{h|A|} \varsigma_{4}, \varsigma_{8}=e^{h|A|} \varsigma_{6}$ and $\varsigma_{9}=e^{h|A|}|B|$ and the simplifying notation $\bar{x}(t)=|x(t)|$ and $S_{\beta}(t)=$

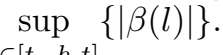

$l \in[t-h, t]$

Then consider the case where $s \in[t-h, t]$. Then, by using the fact that $S_{\beta}(t) \leq S_{\beta}(s)+\sup _{l \in[s, t]}\{|\xi(l)|\}$, from (B.16), it follows that

$$
\begin{aligned}
\bar{x}(t) \leq & \varsigma_{7}(|r(s)|+|\tilde{x}(s)|)+\varsigma_{8} \sup _{l \in[s, t]}\{\psi(l)\} \\
& +\varsigma_{10} S_{\beta}(s)+\varsigma_{9} \sup _{l \in[s, t]}\{|\xi(l)|\}
\end{aligned}
$$

with $\varsigma_{10}=\varsigma_{7}+\varsigma_{9}$. Using (B.15), we obtain, after lengthy but simple calculations,

$$
\begin{aligned}
\bar{x}(t) \leq & \varsigma_{12}|r(s)|+\varsigma_{13} S_{\beta}(s) \\
& +\varsigma_{14}|\tilde{x}(s)|+\varsigma_{11} \sup _{l \in[s, t]}\{\psi(l)\}
\end{aligned}
$$

with $\varsigma_{11}=\varsigma_{9} \varsigma_{6}+\varsigma_{8}, \varsigma_{12}=\varsigma_{7}+\varsigma_{9} \varsigma_{4}, \varsigma_{13}=\varsigma_{10}+\varsigma_{9} \varsigma_{4}$, and $\varsigma_{14}=\varsigma_{7}+\varsigma_{9} \varsigma_{4}$

Next, from the definition of $r$ and the inequality $|\tilde{x}(t)| \leq$ $\bar{x}(t)+|\hat{x}(t)|$, we obtain

$$
\begin{aligned}
\bar{x}(t) \leq & \varsigma_{12}\left(e^{h|A|} \bar{x}(s)+e^{h|A|}|B| S_{\beta}(s)\right) \\
& +\varsigma_{13} S_{\beta}(s)+\varsigma_{14} \bar{x}(s)+\varsigma_{14}|\hat{x}(s)| \\
& +\varsigma_{11} \sup _{l \in[s, t]}\{\psi(l)\} \\
\leq & \varsigma_{15} e^{s-t}\left[\bar{x}(s)+S_{\beta}(s)+|\hat{x}(s)|\right] \\
& +\varsigma_{11} \sup _{l \in[s, t]}\{\psi(l)\},
\end{aligned}
$$

with $\varsigma_{15}=e^{h} \max \left\{\varsigma_{12} e^{h|A|}+\varsigma_{14}, \varsigma_{12} \varsigma_{9}+\varsigma_{13}, \varsigma_{14}\right\}$, where the last inequality is a consequence of $s \in[t-h, t]$.

Now, consider the case where $s<t-h$. Then the last inequality in (B.16) implies that

$$
\begin{aligned}
\bar{x}(t) & \leq \varsigma_{7} e^{-\varsigma_{5}(t-s)}(|r(s)|+|\beta(s)|+|\tilde{x}(s)|)+\varsigma_{8} \sup _{l \in[s, t]}\{\psi(l)\} \\
+\varsigma_{9} & \sup _{m \in[t-h, t]}\left\{\varsigma_{4} e^{-\varsigma_{5}(m-s)}(|\xi(s)|+|\tilde{x}(s)|)+\varsigma_{6} \sup _{l \in[s, m]}\{\psi(l)\}\right\} \\
& \leq \varsigma_{7} e^{-\varsigma_{5}(t-s)}(|r(s)|+|\beta(s)|+|\tilde{x}(s)|) \\
& +\varsigma_{9} \varsigma_{4} e^{-\varsigma_{5}(t-h-s)}(|r(s)|+|\beta(s)|+|\tilde{x}(s)|) \\
& +\varsigma_{8} \sup _{l \in[s, t]}\{\psi(l)\}+\varsigma_{9} \varsigma_{6} \sup _{l \in[s, t]}\{\psi(l)\} .
\end{aligned}
$$

By grouping the terms and using the definition of $r$, we obtain

$$
\begin{aligned}
\bar{x}(t) \leq & \varsigma_{16} e^{-\varsigma_{5}(t-s)}(|r(s)|+|\beta(s)|+|\tilde{x}(s)|) \\
& +\varsigma_{17} \sup _{l \in[s, t]}\{\psi(l)\} \\
\leq & \varsigma_{16} e^{-\varsigma_{5}(t-s)}\left(e^{h|A|} \bar{x}(s)+\varsigma_{9} S_{\beta}(s)+|\beta(s)|+\right. \\
& +\varsigma_{17} \sup _{l \in[s, t]}\left\{\left|\varepsilon\left(g_{2}(l)\right)\right|+|\varphi(l)|\right\} \\
\leq & \varsigma_{18} e^{-\varsigma_{5}(t-s)}\left(e^{h|A|} \bar{x}(s)+\varsigma_{16} S_{\beta}(s)+|\tilde{x}(s)|\right) \\
& +\varsigma_{17} \sup _{l \in[s, t]}\{\psi(l)\},
\end{aligned}
$$

with $\varsigma_{16}=\varsigma_{7}+\varsigma_{9} \varsigma_{4} e^{\varsigma_{5} h}, \varsigma_{17}=\varsigma_{8}+\varsigma_{9} \varsigma_{6}$ and $\varsigma_{18}=e^{h|A|}|B|+$
1. It follows that

$$
\begin{aligned}
\bar{x}(t) \leq & \varsigma_{18} e^{\varsigma_{5}(s-t)}\left(\varsigma_{19} \bar{x}(s)+\varsigma_{16} S_{\beta}(s)+|\hat{x}(s)|\right) \\
& +\varsigma_{17} \sup _{l \in[s, t]}\{\psi(l)\},
\end{aligned}
$$

with $\varsigma_{19}=e^{h|A|}+1$. This inequality and (B.19) allow us to conclude. 